\title{
TERMINOLOGÍA Y TRADUCCIÓN ECONÓMICA FRANCÉS-ESPAÑOL: EVALUACIÓN DE RECURSOS TERMINOLÓGICOS EN EL ÁMBITO CONTABLE
}

\author{
Daniel Gallego Hernández \\ daniel.gallego@ua.es \\ Universidad de Alicante
}

\begin{abstract}
Resumen
El presente trabajo tiene por objetivo evaluar una serie de recursos terminológicos y determinar su adecuación a la práctica de la traducción francés-español de documentos contables. En un primer momento, se identifican las necesidades terminológicas de un colectivo muy concreto de traductores en formación en el ámbito de la traducción económica, así como los recursos que emplean en la práctica de este tipo de traducción, concretamente, en la traducción de cuentas anuales. A continuación, se seleccionan diversos recursos terminológicos utilizados en este contexto y se evalúan conforme a las necesidades previamente detectadas. Los resultados invitan a la elaboración de un diccionario francés-español especializado en el ámbito de la contabilidad y adaptado a las necesidades terminológicas de la práctica de la traducción para este ámbito.
\end{abstract}

\begin{abstract}
"Terminology and French-Spanish business translation: evaluating terminology resources for the translation of accounting documents"

The aim of this paper is to evaluate a series of terminology resources and determine their suitability to the practice of French-Spanish translation of accounting documents. First we identify the terminology needs of a specific group of translator trainees when facing business translation, as well as the resources they use particularly when translating annual accounts. Then, we select various terminology resources used in this context and evaluate them according to their needs. The results suggest the development of a French-Spanish dictionary of accounting terms adapted to the terminology needs of the practice of translation in this area.
\end{abstract}


Palabras clave: Terminología. Traducción económica. Diccionarios especializados. Bases de datos terminológicas.

Keywords: Terminology. Business translation. Specialized dictionaries. Terminology databases. 


\section{Trabajos previos sobre evaluación de recursos}

Productos como los diccionarios han sido desde siempre uno de los principales recursos a los que el traductor de textos especializados, entre ellos, los económicos, acude para satisfacer sus necesidades terminológicas. No cabe duda de que, en la práctica de la traducción económica, estos recursos son una fuente inestimable que ayuda a resolver diferentes problemas y dificultades de traducción. Ahora bien, algunos estudios relacionados con este ámbito dan a entender que no siempre se adecuan a las necesidades del traductor y que, por tanto, pueden presentar igualmente ciertas carencias.

Gerzymish-Arbogast (1989: 193), por ejemplo, entiende que los diccionarios se preocupan más por la cantidad que por la calidad. Consciente de los problemas de ambigüedad y vaguedad que presenta el léxico económico, opina que deberían incluir más información sistemática basada en las relaciones de sentido, que ayude a resolver los problemas de variación terminológica:

An economics dictionary or data bank for translators should focus on providing more text-related information in favour of phonetic, morphological, and syntactic information. As far as macrostructure is concerned, it should be intensive rather than extensive, with a decoding dictionary providing precise definitions and context examples rather than listing a great mass of terms. As far as microstructure is concerned, it should paradigmatically stress lexical information (synonyms, homonyms, polysemes) and above all textual information (relationships between concepts, conditions of use, passepartout quality, and text examples). Syntagmatically, information on the collocation potential and fixed terminological units should be given preference over syntactic information which can be found in other, more general dictionaries.

En consonancia con este trabajo, pero dentro de la combinación italiano-catalán, Camps Olivé (1992: 181) aboga por una mayor calidad informativa y opina que los diccionarios deberían considerar "l'estructura dels camps semàntics, les relacions paradigmàtiques i sintagmàtiques, per tal d'arribar a un instrument de treball que ens informi no tan sols dels sinònims, antònims, hiperònims, homònims i polisèmies, sinó també de les condicions d'ús, i de la collocació". 
En el trabajo de Dancette (1992), quien pone a prueba once diccionarios especializados en el ámbito comercial, económico y financiero a partir de veinte términos problemáticos en traducción inglés-francés, se desprende que el traductor que utiliza diccionarios bilingües puede encontrase con que en el terreno de las equivalencias, o bien no localiza ningún término o expresión, o bien identifica múltiples propuestas de traducción. Este problema parece tener que ver con la dinamicidad del lenguaje comercial y el grado de lexicalización de los términos (cuanto menor es dicho grado mayores problemas se presentan):

La masse des problèmes se situe entre ces deux pôles [termes très lexicalisés et syntagmes ou phrasèmes non lexicalisés] et, malheureusement, à cause de la dynamique du langage des affaires et des nombreux facteurs qui influencent l'usage, on ne peut pas s'attendre à des solutions dictionnairiques simples et rapides. Il faut penser, dans le cas de notions complexes, à intégrer dans une sorte d'algorithme les différentes possibilités de traduction et d'adaptation. (Dancette 1992: 208)

Esta misma autora considera que los diccionarios especializados en el ámbito económico-comercial para traductores y redactores deben ofrecer, además de equivalentes lingüísticos fiables, "une analyse et une description notionnelles telles qu'elles fassent ressortir les concepts clés du domaine et leur agencement à l'intérieur d'une structuration conceptuelle du domaine" (Dancette 1995: 161).

Más recientemente, Fraile Vicente (2007: 13) se refiere a los diccionarios en su relación con la fraseología y pone de relieve la necesidad de que "mejoren su codificación de las expresiones idiomáticas si pretenden responder a las necesidades comunicativas de sus usuarios". Consciente de que no siguen unos criterios claros de inclusión de fraseología y de que no tratan de manera sistemática sus diferentes clases, la autora (2007: 175-182) propone en su obra una codificación de expresiones idiomáticas económicas desde una perspectiva traductológica.

Pero los diccionarios no han sido el único tipo de recursos que se han encontrado en el punto de mira de algunos estudios sobre traducción, también las bases de datos terminológicas han sido objeto de críticas. Por ejemplo, Lerat (1995-1997: 105-106) reconoce que, si bien son útiles para el traductor, "adolecen de limitaciones derivadas de su propia concepción, finalidad, método de ampliación, actualización y depuración”, pues, entre otras cosas, están concebidas para un público concreto, por ejemplo, los servicios comunitarios de Ottawa, en el caso de Termium; están diseñadas por documentalistas, por lo que son prácticamente inexistentes las precisiones semánticas y gramaticales; son semasiológicas (toman como punto de partida formas no 
desambiguadas y lemas) o se alimentan de manera acumulativa, lo que potencia la redundancia de la información.

Por su parte, Tebé Soriano (2001: 204) revela "insuficiencias y contradicciones graves" en el funcionamiento de los bancos de datos terminológicos, relacionadas con la estructuración interna de sus informaciones (diferentes segmentaciones temáticas, falta de relación explícita entre sus registros, variaciones mínimas de significados en los conceptos de áreas próximas) y con su finalidad o adecuación a las necesidades de los usuarios, lo que redunda en una gran cantidad no solo de ruido, sino de silencio en las consultas.

Marcos Mora et al. (2006) consideran que las bases de datos terminológicas plantean dificultades de uso a los traductores en la medida en que no siempre "incorporan aspectos de usabilidad durante el proceso de diseño y desarrollo", pues no parece que existan unas bases consensuadas para el desarrollo de tales aspectos. En este sentido, el trabajo de Marcos Mora y Gómez Martínez (2007) propone una serie de pautas u orientaciones que estas bases de datos podrían considerar con vistas a ser un "buen vehículo de comunicación de información para los usuarios", relacionadas con elementos de navegación interna y externa (enlaces y menús), elementos de comunicación, como los formularios de búsqueda, la disposición gráfica y la redacción de las informaciones o la accesibilidad, entre otros.

De esta breve reseña, en absoluto exhaustiva, de algunos trabajos relacionados directa o indirectamente con los recursos terminográficos en su relación con la práctica de la traducción, puede desprenderse que es conveniente seguir evaluando los recursos que a día de hoy utilizan los traductores, pues algunos de los trabajos mencionados datan de hace ya algunos años. También hemos visto que se trata de estudios referidos a combinaciones lingüísticas específicas (inglés-francés, italiano-catalán, inglés-español). En este sentido, cabe seguir planteándose el tema de la evaluación en otras combinaciones, como francés-español, que es con la que nosotros trabajamos. Asimismo, los comentarios en torno a tal o cual diccionario no hacen alusión a ninguna área concreta de la economía y de los negocios ni a ningún tipo de encargo, lo que nos ha hecho pensar que la evaluación de este tipo de recursos puede centrarse en alguno de estos dos últimos aspectos. Respecto de los trabajos en torno a las bases de datos terminológicas, algo más recientes que los anteriores, se trata de estudios no referidos al ámbito de la economía y los negocios, sino, más bien, referidos a su macro y microestructura (usabilidad, adecuación a las necesidades de usuarios, relaciones entre campos, etc.). En este sentido, consideramos que podemos estudiar la utilidad de alguna de dichas bases en relación igualmente con algún aspecto concreto de la economía. 
Acabamos, pues, de constatar que todavía cabe seguir evaluando los recursos terminológicos de los que dispone el traductor y hemos enfatizado la necesidad de enmarcar dicha evaluación en determinados contextos de trabajo. En este sentido, a continuación, trazamos el diseño que hemos seguido para contextualizar la evaluación de una serie de recursos terminológicos. En concreto, presentamos, en un primer momento, el colectivo de usuarios de dichos recursos y sus condiciones de trabajo. En un segundo momento, recapitulamos las necesidades terminológicas del colectivo seleccionado y reseñamos el tipo de recursos que utilizan a la hora de enfrentarse a un encargo de traducción determinado. Todo ello, con el fin de seleccionar una serie de recursos terminológicos actuales para evaluarlos en función de la combinación lingüística del encargo, así como en relación al área de especialización en cuestión.

\section{Colectivo y contexto de trabajo}

El colectivo con el que trabajamos en el presente estudio es el de traductores en formación del Máster de Traducción Institucional de la Universidad de Alicante. Se trata de un máster on line con carácter profesionalizante cuyo plan de estudios se distribuye básicamente en dos grandes módulos: uno común, en el que los traductores en formación cursan asignaturas como Corrección y edición profesional de textos y Deontología y práctica profesional, y otro especializado ofrecido para tres ramas lingüísticas (alemán, francés e inglés), formado por varias asignaturas: Ordenamientos jurídicos comparados, Traducción jurada, Traducción para los organismos internacionales y Traducción para el ámbito económico y comercial. Aparte de estas, el traductor en formación tiene la posibilidad de cursar otras asignaturas optativas, como Traducción para la propiedad intelectual, Traducción para la exportación y el comercio exterior o Interpretación para los organismos internacionales, entre otras.

El presente estudio se enmarca concretamente dentro de la asignatura Traducción para el ámbito económico y comercial, en la rama lingüística francésespañol y español-francés. Se trata de una asignatura en la que se trabajan diferentes géneros textuales relacionados con la economía y los negocios y se aplica una metodología de trabajo variada (análisis discursivo de textos, estudio de la terminología y fraseología bilingüe de los textos, explotación de textos paralelos y corpus, utilización de recursos terminológicos). Todo ello, con el apoyo de una plataforma virtual de trabajo que permite no solo la publicación de materiales, la entrega y corrección de trabajos (envío de archivos, ejercicios de tipo test, etc.), sino también la interacción en foros de debate tanto entre el formador y los propios traductores como entre ellos mismos. 
El colectivo que cada año cursa esta asignatura suele ser variado, de edades muy diversas y procedencias distintas: estudiantes recién licenciados en Traducción e Interpretación en la Universidad de Alicante o en otras universidades, estudiantes recién licenciados en otros estudios, estudiantes francófonos procedentes de universidades extranjeras, estudiantes que ya ejercen como traductores autónomos, etc. A pesar de esta heterogeneidad, quizá sea posible emparentar este colectivo con el perfil del traductor autónomo generalista que comienza su andadura por el mundo profesional y que, en materia de traducción económico-financiera, debe adquirir ad hoc los conocimientos necesarios para llevar a cabo una traducción aceptable.

\section{Necesidades terminológicas en el marco de la traducción económica}

Diversos autores se han preocupado por resaltar la importancia de la documentación en traducción económica. Mayoral Asensio (1996) propone un modelo que combina el uso de fuentes textuales (textos multilingües, paralelos, auténticos, extraídos de formularios, redactados en su forma original, traducidos y próximos) y fuentes léxicas. Fuertes-Olivera (2003) sugiere que los traductores sin conocimientos especializados consulten manuales, monografías y diccionarios especializados para elaborar resúmenes de los originales a los que se enfrentan, y, con ello, conocer los conceptos y denominaciones del campo especializado en ambas lenguas. Socorro Trujillo (2004) propone el empleo de diversos tipos de recursos informativos, así como un estudio textual comparado con la ayuda de textos paralelos y próximos. Heras Díez (2005: 21) considera que la documentación debe beneficiarse de los servicios que ofrece internet, pues "los diccionarios monolingües o bilingües se quedan cortos". Lobato Patricio et al. (2010) plantean una metodología docente y sugieren la consulta de diccionarios monolingües generales y especializados, sitios webs de organismos internacionales, sitios de empresas aludidas en los textos originales, etc. Gallego Hernández (2012) propone una metodología basada en la explotación de textos paralelos y corpus en su aplicación a la traducción económica.

Independientemente del proceso o estrategia de documentación que siga el traductor, el resultado del proceso tiene que ver, en esencia, con la resolución de problemas y dificultades relacionados con la terminología, elemento clave en traducción especializada. Los problemas terminológicos básicos a los que el traductor suele enfrentarse pueden resumirse en cuatro, tal como propone Cabré (2010: 359):

Not knowing all or part of a term, its meaning, its grammatical use or pragmatic value in the source language. / Not knowing if in the target language 
there is a lexicalized unit semantically and pragmatically equivalent to the term used in the original text. / Doubting whether a given unit of the target language is the most appropriate equivalent among the alternatives found. / Ignoring or having doubts about the phraseology used in a particular field of speciality.

En cuanto a este último punto relacionado con la fraseología, según los expertos en traducción especializada, los problemas que genera este tipo de unidades no suelen ser tanto de comprensión o identificación, sino de reexpresión, pues, con frecuencia, su reformulación requiere una cadena de caracteres que se adecue a la estereotipia y a las características propias del lenguaje de especialidad de la lengua de llegada (Gouadec 1997: 175; Gómez González-Jover 2007: 34). Esta realidad también afecta, por supuesto, a la traducción económico-financiera: Durban (2005: 66), por ejemplo, menciona la necesidad de buscar "le ton" en la traducción para satisfacer las expectativas de los clientes, en clara alusión a la necesidad de reproducir en la lengua de llegada un lenguaje similar al de los especialistas.

Este tipo de problemas y dificultades se da realmente en el contexto formativo dentro del que se enmarca el presente estudio, al menos si tenemos en cuenta las dudas que los traductores en formación suelen plasmar en uno los foros de debate dispuestos en la asignatura, destinado específicamente a la resolución de problemas de traducción. Veamos ejemplos de las necesidades que se desprenden de algunas intervenciones:

El término "PIB real" viene definido de la siguiente manera: 1) por la Caixa: PIB expresado en euros constantes, tomando como referencia un año determinado [... 2) en una página web colombiana: mide la actividad económica de un país a precios constantes, es decir, revela los cambios en la producción económica una vez realizados los ajustes por inflación [...] 3) en EUMED: PIB real (o a precios constantes): es el valor monetario de todos los bienes y servicios de consumo final producidos por una economía durante un periodo determinado de tiempo (un trimestre, un semestre o un año), calculado utilizando los precios de mercado de un periodo base fijo [...] He encontrado tres equivalentes en francés en el diccionario económico DICOTREC: PIB réel; PIB en volume; PIB en valeur. En el IATE aparecen "PIB en volume" y "PIB en termes réels". Si miramos las definiciones francesas para PIB réel y PIB en volume, obtenemos: "Le produit intérieur brut réel, ou produit intérieur brut en volume, est une mesure du produit intérieur brut basée sur la variation du PIB en volume d'une période de référence à une autre (généralement une année ou un trimestre), mesuré avec des prix constants [...] Al comparar informes del BCE en español y francés, he encontrado PIB réel y PIB en volume" [...] Al comparar informes anuales del BCE en español y en francés, encuentro estos dos términos para PIB real así que pienso que se pueden 
poner los dos. ¿Qué pensáis? ¿Alguien sabe si hay que poner uno u otro en función de otros datos?

Esta intervención deja patente la necesidad terminológica que tiene este traductor en formación como consecuencia de la variación terminológica. Efectivamente, además de tener que construir el concepto PIB real a partir de diferentes definiciones tanto en español como en francés, le surge la duda en torno al uso de dos denominaciones en francés, que persiste incluso después de haber buscado no solo en fuentes terminográficas, sino también en textos paralelos.

Me surge una duda con "inversión en otras construcciones" ya que no entiendo a qué se refiere exactamente. En Francia tenemos "les constructions résidentielles" y "les constructions non résidentielles / hors logements". ¿A esto se refiere?

He aquí otra expresión simpática: "à parc comparable". Para ella solo encuentro textos paralelos, pero no consigo descifrar lo que quiere decir. Lo más cerca que he llegado ha sido con IATE, que para "parc de biens de capital fixe" nos da "stock de bienes de capital fijo".

En estos otros dos casos, en cambio, la necesidad tiene que ver, más bien, con la comprensión de unidades especializadas. En el primer ejemplo, el traductor en formación trata de resolver su duda instando a sus compañeros a que se pronuncien en torno a una hipótesis de traducción. En el segundo ejemplo, se aprecia claramente que el traductor no consigue comprender el término y que ha acudido a IATE, lo que parece haberle alejado de su significado.

¿Deberíamos hacer una traducción literal de "multiformat" o encontrar otro término?

Este caso, en cambio, tiene que ver con necesidades relacionadas con la identificación de equivalentes. El traductor en formación entiende el término, pero duda entre hacer una traducción literal o dedicar su tiempo a buscar un término equivalente en lengua de llegada.

"Escalado de intereses por tramos", me he quedado un poco bloqueada en este término, si alguien me puede ayudar...

Este último ejemplo afecta, más bien, a las necesidades referidas a la reexpresión de fraseología, pues el significado puede deducirse del contexto. En concreto, el traductor se enfrenta a una expresión que no aparece en los recursos a la que debe buscar una propuesta de traducción.

Como puede apreciarse de estos ejemplos extraídos del foro utilizado para la resolución de dudas terminológicas, se aprecia que las necesidades que tiene el traductor en formación son de diversa naturaleza y que no siempre los 
recursos que utiliza el traductor en su proceso de documentación le ayudan a satisfacerlas de manera eficiente.

\section{Encargo de traducción: traducción de cuentas anuales del francés al español}

Hasta el momento, hemos descrito el colectivo con el que trabajamos para este estudio, así como algunas de sus necesidades terminológicas que, como hemos anticipado, no siempre terminan por resolverse con recursos terminológicos. Para evaluar de manera un tanto más sistemática este tipo de recursos, partimos concretamente de los trabajos finales de la asignatura mencionada, entregados durante los tres últimos cursos académicos (treinta estudiantes en total). Se trata de un trabajo que insta a los traductores en formación a enfrentarse a la simulación de un encargo real, como si fueran traductores autónomos. En él, además de llevar a cabo la traducción de un documento de mayor extensión que los vistos durante el curso, también debieron explicitar en un informe complementario, entre otras cosas, el proceso de documentación que siguieron, los recursos consultados, así como los problemas y dificultades de traducción a los que tuvieron que enfrentarse.

En concreto, los traductores tuvieron que verter al español unas cuentas anuales elaboradas en francés. Se trata de uno de los documentos contables por excelencia. La contabilidad puede entenderse como "un sistema de información destinado a transformar datos económicos y financieros en información útil para que sus usuarios puedan basar en ella la toma de sus decisiones económicas" (Solà Tey \& Vilardell Riera 2009: 29). Entre otras cosas, es posible hablar de diferentes tipos de contabilidad:

- macrocontabilidad, generalmente elaborada por los Estados, y microcontabilidad, elaborada por unidades o agentes económicos de menor tamaño;

- contabilidad pública, llevada a cabo por administraciones públicas, y privada, orientada a las organizaciones empresariales;

- contabilidad interna o contabilidad para la gestión, referida al ámbito interno empresarial de utilidad para la optimización de la toma de decisiones, y contabilidad externa, llamada igualmente contabilidad financiera, elaborada para accionistas, directivos, acreedores, proveedores, trabajadores, clientes, etc., de modo que conozcan las transacciones que se dan entre la organización y su entorno;

- contabilidad semestral, la elaborada cada semestre, y contabilidad anual, la elaborada anualmente, por lo general, con carácter obligatorio. 
Dentro de esta clasificación, el documento que propusimos como encargo de traducción, puede enmarcarse en el ámbito de la microcontabilidad privada externa anual, es decir, en la contabilidad llevada a cabo en el seno de sociedades con ánimo de lucro que tiene el propósito de reflejar su situación financiera a finales de año, de modo que los usuarios externos, como propietarios que encomiendan a administradores la gestión de la sociedad, entidades financieras, acreedores comerciales, organismos estatales o analistas y asesores financieros puedan conocer dicha situación para, respectivamente, decidir la conveniencia de invertir en la sociedad sus recursos o liquidar su participación, aceptar o no las operaciones financieras solicitadas por la sociedad, evaluar la capacidad de los proveedores de cumplir con posibles acuerdos, establecer medidas de política económica o informar y asesorar en materia de inversión.

Se trata de un documento que, según el Plan Comptable Général de Francia, está integrado por tres documentos básicos:

- le bilan (el balance), que tiene por objetivo presentar de manera sintética las cuentas patrimoniales de la empresa: se trata de una tabla que contempla el activo, el pasivo y el patrimonio neto de la sociedad;

- le compte de résultat (la cuenta de pérdidas y ganancias), que recoge el resultado del ejercicio sobre el que versan las cuentas: permite evaluar la eficiencia de la empresa contrastando los ingresos y los gastos del ejercicio, también de manera sintética en una tabla;

- l'annexe (la memoria), que complementa, amplía y explica el contenido de los documentos anteriores: se trata de un documento que, si bien no tiene una estructura fija, suele contener una información mínima en torno a la actividad de la empresa, bases de presentación de resultados, normas de valoración, etc.

Según el Nuevo Plan General de Contabilidad español de 2007, las cuentas anuales están integradas por cinco documentos: además del balance, la cuenta de pérdidas y ganancias y la memoria, se encuentra el estado de cambios en el patrimonio neto, cuyo objetivo es mostrar cómo cambia el patrimonio de la empresa de un ejercicio a otro, y el estado de flujo de efectivos, que muestra los pagos y cobros producidos en un ejercicio (la caja).

Se trata, pues, de un documento que contiene abundante terminología, en especial dentro del balance y la cuenta de pérdidas y ganancias, pero también fraseología especializada, concretamente dentro de la memoria, pues, a diferencia de los otros dos documentos, es un texto redactado. En definitiva, es un documento cuya área de especialización no suele ser conocida por los traductores en formación, quienes, en consecuencia, necesitan invertir cierta 
parte de su tiempo para satisfacer las diferentes necesidades terminológicas relacionadas tanto con los conceptos como con las denominaciones en la lengua de llegada. El documento real que tuvieron que traducir parcialmente se encuentra alojado en: http://www.info-financiere.fr/upload/CNS/8888/01/ FCCNS025218_20100209.pdf.

\section{Recursos empleados en el encargo de traducción por el colectivo analizado}

Son diversos los tipos de recursos que pueden utilizarse para resolver tal o cual problema o dificultad de corte terminológico. Según Gallego Hernández (2012: 122-125), es posible distinguir tres tipos de recursos o fuentes primarias básicos: expertos o fuentes humanas; fuentes temáticas, conocidas en los estudios de traducción como textos paralelos, los cuales, en esencia, comparten algún aspecto de la situación comunicativa del texto original; y, por último, fuentes lingüísticas, es decir, materiales de referencia elaborados básicamente con el propósito de proporcionar información lingüística específica relacionada con la lengua: diccionarios, glosarios, bases de datos terminológicas, etc. Según esta clasificación y los informes entregados, los recursos que usaron los traductores en su encargo fueron los siguientes.

Respecto de las fuentes humanas, además del foro de debate para la resolución de problemas de traducción de la propia asignatura, los traductores también acudieron a foros externos. Entre los indicados en sus informes, figuran foros de traductores, como ProZ (Freelance translators $\&$ Translation companies) o Yahoo, además de los de Wordreference.

Por lo que se refiere a las fuentes temáticas, reseñaron, entre otras, el sitio europeo sobre las Normas internacionales de contabilidad (NIC), que posibilita la consulta de diferentes normas y directivas en varios idiomas en materia de contabilidad empresarial; la legislación de la Unión Europea (EurLex), disponible en sus diferentes lenguas oficiales, que supone una fuente inagotable de textos paralelos de verdadera utilidad para la traducción, ya no solo para el ámbito de la contabilidad, sino de muchos otros; el sitio de la Comisión Nacional del Mercado de Valores (CNMV), que contiene múltiples informes anuales de diferentes organizaciones que, a su vez, contienen documentación contable en forma de cuentas anuales. La consulta de este tipo de sitios se vio complementada con la búsqueda de portales especializados en la materia, así como de organizaciones concretas que previsiblemente publican el mismo tipo de texto al que se enfrentaron los traductores: diversos sitios de organizaciones, como Mercadona e Iberdrola, con el propósito de obtener, tal como en el caso anterior, informes de auditoría y cuentas anuales, así como 
diversos portales especializados en economía y derecho, como Actufinance (Les coulisses de la finance), Le droit sur internet o los planes generales de contabilidad en ambos idiomas.

Por último, en cuanto a las fuentes lingüísticas, los traductores emplearon igualmente diversos tipos de recursos que van de lo general a lo especializado, tanto monolingües (en la lengua original o de llegada), como multilingües. En este sentido, además de diferentes diccionarios generales, como los de Wordreference, Wikipedia o Reverso, usaron diccionarios especializados bilingües, como Dicotrec (Diccionario para traducción económica), Diccionario LID multilingüe de la empresa y economía, de Marcelino Elosua, o el Diccionario jurídico-económico: francés-español, español-francés, de Nicolás Campos et al. Asimismo, hicieron uso de diccionarios especializados monolingües tanto en lengua de llegada como en lengua original: premysa.org/pdf/emprendedores/ GlosarioEconomico-Financiero.pdf, www.economia48.com, www.eco-finanzas.com/conceptos.htm o www.edubourse.com/lexique/rnpg.php, así como de bases de datos terminológicas, como IATE (el recurso más mencionado en los informes), Termcat o UNTERM. Siguiendo las tareas exigidas en el encargo, también elaboraron su propia herramienta ad hoc en forma de corpus a partir de textos paralelos recuperados de la web, tal como se les sugirió a lo largo de la asignatura, para proceder posteriormente a su explotación off line en su estación de trabajo con la herramienta Antconc.

Se trata de una selección de recursos que en absoluto es exhaustiva, pues los traductores en formación detallaron en sus trabajos diferentes enlaces a páginas y documentos mucho más concretos, algunos de los cuales ya no se encuentran disponibles en la actualidad. En cualquier caso, se trata de una selección que, a nuestro entender, además de bastarse para tratar de llevar a cabo el presente estudio de caso, refleja de manera global el tipo de fuentes que utiliza este colectivo e incluso ofrece pistas sobre su manera de actuar.

\section{Evaluación de recursos terminológicos empleados: análisis de las fuentes lingüísticas}

De los tipos de fuentes previamente reseñados, se puede afirmar, sin entrar en detalle, que las fuentes personales tienen la ventaja de que pueden ayudar a satisfacer diversas necesidades y que es posible consultarlas con lenguaje natural, aunque de manera puntual, sin hacer un abuso de ellas (el traductor corre el riesgo de no volver a obtener respuesta si interviene constantemente en los foros solo para plantear dudas). En cuanto a las fuentes temáticas, las escogidas por los traductores en formación parecen ser muy acertadas. Sin embargo, el problema que presentan tiene que ver, en primer lugar, con su 
localización, y, en segundo lugar, con su consulta. Efectivamente, identificar una fuente temática de validez pasa inevitablemente no solo por una evaluación previa, sobre todo si se desconoce el tema, sino también por acceder a la información exacta que se precisa, que no siempre se encuentra en el documento, lo que puede suponer una importante inversión de tiempo (en especial si se decide compilar un corpus ad hoc y no se tienen desarrolladas adecuadamente las competencias tecnológicas y especializadas para optimizar el proceso). En cualquier caso, este tipo de fuentes puede ayudar a ver cómo los nativos especialistas de la lengua de llegada emplean tal o cual unidad terminológica o fraseológica, lo que, a su vez, permite que el traductor redacte su traducción de manera similar a como se expresan, sin necesidad de formar parte de este colectivo.

Respecto de las fuentes lingüísticas, concretamente las terminológicas, teniendo en cuenta el objetivo del presente estudio, analizaremos una serie de recursos concretos. Estudiaremos, por una parte, las bases de datos terminológicas IATE, Cercaterm (Termcat) y UNTERM, pues son los recursos a los que los traductores en formación más aludieron en sus informes. Por otra parte, estudiaremos los diccionarios Dicotrec, un recurso en línea elaborado en 2007 y 2008 por traductores en formación en el marco de la asignatura Traducción Especializada A-B y B-A de $4^{\circ}$ curso de la Licenciatura de Traducción e Interpretación de la Universidad Autónoma de Madrid, el Diccionario jurídico-económico: francés-español, español-francés, de Nicolás Campos et al. (2005), y la $11^{a}$ edición del Diccionario LID multilingüe de la empresa y economía, de Marcelino Elosua (2007), estos dos últimos, en formato papel. Se trata de diccionarios actuales especializados en economía también mencionados en los informes. Nos centraremos tanto en el análisis de sus propuestas de traducción como en la información microestructural de sus entradas, además de comentar su modo de empleo, siempre en relación con el encargo determinado: traducción francés-español de cuentas anuales.

Para evaluar las propuestas de traducción de las bases de datos, interrogamos sus interfaces con los trigramas más frecuentes que la estación de trabajo Terminus ${ }^{1}$ nos devolvió al analizar el texto original al que se enfrentaron los traductores y que reproducimos a continuación:

1. Terminus es una estación de trabajo diseñada por el Institut Universitari de Lingüística Aplicada de la Universitat Pompeu Fabra para la terminología. Permite llevar a cabo la cadena completa del trabajo terminográfico: creación y explotación de corpus, gestión de glosarios y proyectos, creación y mantenimiento de bases de datos y edición de diccionarios. 




La siguiente tabla muestra las propuestas de traducción que ofrecen las tres bases de datos para cada una de las unidades escogidas (eliminamos del listado anterior los trigramas referidos a nombres propios y las variantes formales de género y número):

\begin{tabular}{|l|l|l|l|}
\hline \multicolumn{1}{|c|}{ UNIDAD } & \multicolumn{1}{c|}{ IATE } & \multicolumn{1}{c|}{ TERMCAT } & \multicolumn{1}{c|}{ UNTERM } \\
\hline flux de trésorerie & flujo de tesorería & flujo de caja & corriente de efectivo \\
\hline $\begin{array}{l}\text { équivalents de } \\
\text { trésorerie }\end{array}$ & \multicolumn{1}{c|}{- --- } & $\begin{array}{l}\text { equivalentes al } \\
\text { efectivo }\end{array}$ \\
\hline $\begin{array}{l}\text { amortissements et } \\
\text { dépréciations }\end{array}$ & $\begin{array}{l}\text { [hay que buscar un } \\
\text { término y luego el } \\
\text { otro] }\end{array}$ & $\begin{array}{l}\text { [hay que buscar un } \\
\text { término y luego el } \\
\text { otro] }\end{array}$ & $\begin{array}{l}\text { [hay que buscar un } \\
\text { término y luego el } \\
\text { otro] }\end{array}$ \\
\hline compte de résultat & $\begin{array}{l}\text { cuenta de } \\
\text { resultados; } \\
\text { cuenta de pérdidas y y } \\
\text { ganancias }\end{array}$ & $\begin{array}{l}\text { cuenta de } \\
\text { explotación; cuenta } \\
\text { de pérdidas y } \\
\text { ganancias; cuenta de } \\
\text { resultados }\end{array}$ & estado de ingresos \\
\hline impôts différés & impuestos diferidos & impuestos diferidos & \multicolumn{2}{|l}{--- } \\
\hline
\end{tabular}




\begin{tabular}{|c|c|c|c|}
\hline produits et charges & $\begin{array}{l}\text { ingresos y gastos; } \\
\text { gastos e ingresos; } \\
\text { rendimientos y } \\
\text { cargos; costes y } \\
\text { productos; cargas y } \\
\text { productos; }\end{array}$ & ingresos y gastos & $\begin{array}{l}\text { [hay que buscar un } \\
\text { término y luego el } \\
\text { otro] }\end{array}$ \\
\hline emprunts et dettes & $\begin{array}{l}\text { préstamos y deudas; } \\
\text { empréstitos y } \\
\text { deudas }\end{array}$ & $\begin{array}{l}\text { [hay que buscar un } \\
\text { término y luego el } \\
\text { otro] }\end{array}$ & $\begin{array}{l}\text { [hay que buscar un } \\
\text { término y luego el } \\
\text { otro] }\end{array}$ \\
\hline chiffre d'affaires & $\begin{array}{l}\text { volumen de } \\
\text { negocios; cifra de } \\
\text { negocios }\end{array}$ & $\begin{array}{l}\text { facturación; } \\
\text { volumen de negocio }\end{array}$ & --- \\
\hline charge d'impôt & $\begin{array}{l}\text { carga fiscal; carga } \\
\text { tributaria }\end{array}$ & --- & --- \\
\hline pertes de valeur & pérdida en valor & --- & --- \\
\hline $\begin{array}{l}\text { [flux] net de } \\
\text { trésorerie }\end{array}$ & --- & --- & --- \\
\hline plan de sauvegarde & $\begin{array}{l}\text { plan de } \\
\text { recuperación }\end{array}$ & --- & --- \\
\hline valeur d'utilité & --- & --- & --- \\
\hline
\end{tabular}

tabla 1

Como puede apreciarse en la tabla, son múltiples los casos que se dan:

- ninguna base ofrece ninguna propuesta, caso de valeur d'utilité o flux net de trésorerie;

- alguna de las bases no ofrece ninguna propuesta, casos de équivalents de trésorerie, impôts différés, perte de valeur o plan de sauvegarde;

- cada una de las bases ofrece una propuesta de traducción distinta, caso de flux de trésorerie;

- dos o más bases ofrecen diferentes variantes denominativas coincidentes entre ellas, casos de compte de résultat;

- dos o más bases ofrecen diferentes variantes denominativas que no coinciden entre ellas, ya sea por cambios léxicos o variantes formales, casos de compte de résultat o chiffre d'affaires;

- solo una base ofrece propuestas de traducción, pero contiene variaciones denominativas, caso de charge d'impôt o emprunts et dettes;

- en el caso de las unidades coordinadas por la conjunción et, algunas bases recuperan resultados cuando se les interroga con la construcción en cuestión.

En cuanto a las posibilidades de consulta, las tres bases de datos funcionan por medio de la introducción de palabras clave, que pueden filtrarse según los 
campos que hayan sido diseñados en cada una de ellas, básicamente, idioma y campo de especialidad, aunque, dependiendo del sistema, también es posible filtrar las búsquedas a los campos de determinados datos, como definiciones o notas, caso de TERMCAT. El sistema recupera una serie de resultados que responden a la búsqueda establecida. Estos resultados no se muestran a modo de ficha, sino que se trata, más bien, de una especie de descriptores o fichas sinópticas correspondientes a diferentes entradas que guían al usuario. Este último tiene la posibilidad de navegar por cada resultado y acceder a la ficha terminológica correspondiente a cada entrada. Las tres bases de datos también permiten atribuir condiciones o comodines a las palabras clave que se pretenden buscar. IATE y UNTERM, por ejemplo, permiten el empleo de comodines como *, que busca cualquier secuencia de caracteres. TERMACT, por su parte, permite hacer búsquedas aproximadas, que comiencen o acaben por tal secuencia de caracteres, por ejemplo.

Respecto de las propuestas de traducción de los diccionarios especializados, buscamos algunos de los primeros términos que la estación de trabajo Terminus nos devolvió al utilizar su herramienta de extracción terminológica y que reproducimos a continuación:

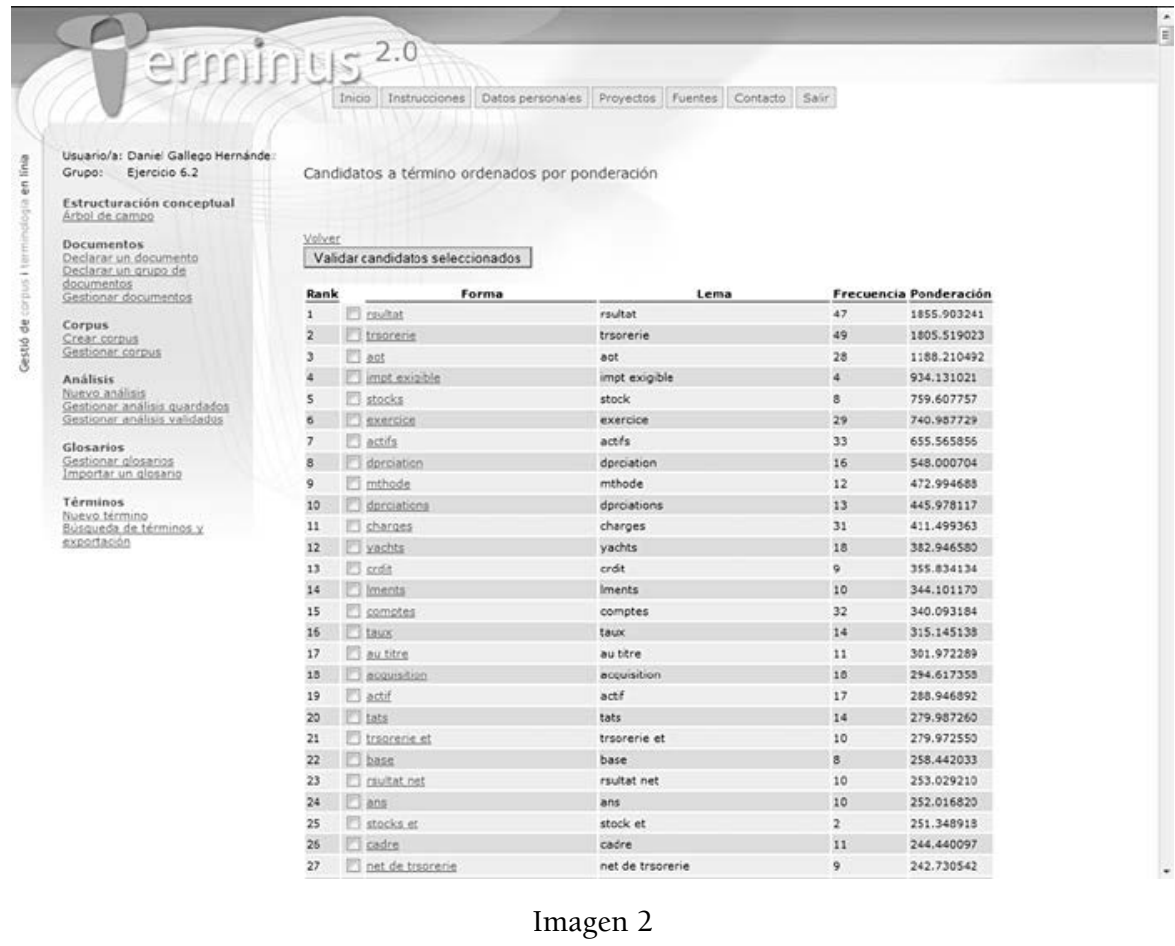


Sin entrar a valorar la pertinencia de los candidatos a término extraídos ni la de la elaboración del diccionario de entrenamiento utilizado para su extracción, ${ }^{2}$ creemos que los resultados que ofrece Terminus suponen una base suficiente de elementos terminológicos con los que proceder a evaluar las propuestas de traducción que ofrecen los diccionarios especializados escogidos, recordemos, Dicotrec, el Diccionario jurídico-económico: francés-español, español-francés, y el Diccionario LID multilingüe de la empresa y economía:

\begin{tabular}{|c|c|c|c|}
\hline UNIDAD & DICOTREC & CAMPOS & LID \\
\hline résultat & \begin{tabular}{|l} 
[aparece en \\
compuestos]
\end{tabular} & resultado & $\begin{array}{l}\text { puntuación, resultados } \\
\text { [résultats] }\end{array}$ \\
\hline trésorerie & tesorería & $\begin{array}{l}\text { tesorería, hacienda } \\
\text { pública }\end{array}$ & $\begin{array}{l}\text { tesorería, dinero } \\
\text { circulante }\end{array}$ \\
\hline impôt exigible & $\begin{array}{l}\text { [hay que buscar } \\
\text { un término y } \\
\text { luego el otro] }\end{array}$ & $\begin{array}{l}\text { [hay que buscar un } \\
\text { término y luego el } \\
\text { otro] }\end{array}$ & impuesto exigible \\
\hline stocks & existencias & stock, existencias & existencias [stock] \\
\hline exercice & ejercicio & ejercicio & ejercicio \\
\hline actifs & activo & activo & $\begin{array}{l}\text { recursos [actifs]; activo } \\
\text { [actif] }\end{array}$ \\
\hline dépréciation & --- & depreciación & depreciación, desgaste \\
\hline méthode & \begin{tabular}{|l}
$\begin{array}{l}\text { método } \\
\text { (contable) }\end{array}$ \\
\end{tabular} & --- & método \\
\hline charges & gastos & carga; cargo; gastos & gravamen [charge] \\
\hline crédit & crédito & crédito & haber, crédito \\
\hline éléments & --- & factor; elemento & \begin{tabular}{|l} 
[aparece en \\
compuestos]
\end{tabular} \\
\hline comptes & cuenta & cuenta & cuentas \\
\hline $\operatorname{taux}$ & $\begin{array}{l}\text { [aparece en } \\
\text { compuestos] }\end{array}$ & tipo, tasa & $\begin{array}{l}\text { cuota [también aparece } \\
\text { en compuestos, } \\
\text { pero con otras } \\
\text { denominaciones] } \\
\end{array}$ \\
\hline au titre & --- & $\begin{array}{l}\text { en concepto de; de } \\
\text { acuerdo con; con } \\
\text { arreglo a [au titre de] } \\
\end{array}$ & --- \\
\hline
\end{tabular}

Tabla 2

2. Para hacer funcionar la herramienta de extracción de términos fue preciso entrenar a Terminus introduciendo un diccionario especializado con al menos 500 entradas en francés. Para confeccionarlo, acudimos a los resultados que nos ofreció Google al interrogarlo con la expresión "glossaire comptabilité". Conseguimos elaborar un glosario ad hoc a partir de los siguientes sitios: www.bizattitude.ch/CMS/default.asp?ID=211, www.lacompta.org/ vocabulaire/index.php, www.e-conomic.fr/logiciel/termes-comptables, www.becompta. be/modules/dictionnaire/ y www.lexique-comptable.info/lcab.html. 
En los resultados expuestos en esta tabla podemos apreciar lo siguiente:

- los diccionarios no siguen los mismos criterios a la hora de lexicalizar tal o cual unidad;

- los diccionarios, en ocasiones, coinciden de lleno en sus propuestas de traducción, caso de exercice o comptes;

- coinciden en su primera propuesta de traducción, pero no en las variantes denominativas, caso de trésorerie;

- no incluyen en una entrada la unidad con la que los hemos interrogado, pero sí aparece en otras entradas como una unidad compuesta, caso de taux o résultat en DICOTREC;

- la propuesta de traducción que ofrecen para la unidad en cuestión no coincide con otras propuestas cuando dicha unidad aparece en unidades compuestas, caso de taux en LID;

- la propuesta de traducción para alguna unidad varía dependiendo del número, casos de actifs y charges (en compuestos) en LID;

- hay que buscar una propuesta de traducción para cada uno de los elementos que componen la unidad, caso de impôt exigible, en DICOTREC y CAMPOS;

- pueden llegar a lexicalizar locuciones de tipo preposicional, como au titre de en CAMPOS.

Por lo que se refiere al modo de empleo de estos diccionarios, el proceso es el típico de un diccionario en papel: la ordenación alfabética propuesta en la macroestructura de estos recursos permite al usuario acceder a tal o cual unidad, así como a sus respectivas informaciones. En el caso del diccionario LID, al tratarse de un recurso de más de dos lenguas, se incluye al final de la obra diferentes listados bilingües en distintas combinaciones lingüísticas, entre ellas el francés-español, cuyas propuestas pueden consultarse en las entradas en español, que contienen las definiciones. DICOTREC, sin embargo, a pesar de ser un recurso electrónico en línea, no permite la opción de que el recurso busque el término introducido en la casilla de búsqueda si no es por medio de la opción de búsqueda del propio navegador.

Hasta el momento, hemos estudiado una serie de propuestas de traducción y comentado los modos de empleo tanto de las bases de datos terminológicas como de los diccionarios especializados escogidos. Interroguémonos ahora, aunque de manera muy breve, por otro de los elementos microestructurales de los recursos estudiados, en concreto, por la fraseología especializada. Pongamos, por ejemplo, las siguientes cadenas aparecidas en el texto original del encargo: 
- flux de trésorerie liés aux opérations

- génèrent pas de flux de trésorerie

- produits et charges sont entièrement intégrés

Se trata de tres unidades fraseológicas que aparecen no solo en el texto del encargo, sino también, con frecuencia, en textos de contabilidad en francés. Si bien es posible consultar las unidades terminológicas (no se encuentran registradas como entradas en ninguno de los recursos) que aparecen dentro de estas cadenas en los diferentes recursos identificados en el presente estudio, lo cierto es que ninguno de ellos ofrece información bilingüe en torno al comportamiento discursivo de las unidades en cuestión. Dicho de otro modo, es posible buscar flux de trésorerie u opération, flux de trésorerie o générer, produits et charges o intégrer, pero los resultados que podemos obtener no relacionan entre sí las unidades buscadas. En consecuencia, una de las necesidades terminológicas reseñadas anteriormente, la relacionada con la fraseología, puede no verse satisfecha, si no es complementándose con otro tipo de fuentes, como los textos paralelos.

Los resultados de estos análisis muestran que algunos de los problemas detectados en el marco de la terminología contable francés-español se corresponden efectivamente con los reseñados, de manera general, por el grupo IULATERM (2013):

no saber si la lengua meta (LM) dispone de una unidad terminológica lexicalizada para expresar una idea que en la lengua de partida (LP) se expresaba mediante un término porque ésta no figura en los diccionarios especializados / no saber si las unidades que les ofrecen los diccionarios bilingües especializados son las más adecuadas para el texto de traducción / no saber qué unidad deben seleccionar cuando las fuentes terminológicas ofrecen diferentes alternativas

En efecto, hemos apreciado que, por una parte, el traductor en formación puede toparse con ruido informativo, especialmente cuando los recursos ofrecen diferentes variantes denominativas como propuesta de traducción para un mismo término, exentas, la mayor parte de las veces, de comentarios que le ayuden a tomar una decisión, por ejemplo, en torno a la variación dialectal que pueda existir. Ello le lleva a tener que contrastar las propuestas identificadas en otras fuentes, ya sean lingüísticas, ya sean temáticas o personales. Por otra parte, el traductor en formación también puede toparse con silencio, lo que le supone llevar a cabo una nueva búsqueda en otros recursos. Este problema del silencio parece acentuarse en el caso de la traducción de la fraseología especializada. 


\section{Propuesta de mejora de los recursos existentes}

De lo expuesto hasta ahora se desprende que, si bien la totalidad de recursos utilizados, ya sean expertos, ya sean textos paralelos, ya sean recursos lingüísticos, puede ayudar a elaborar una traducción aceptable, lo cierto es que los recursos terminológicos especializados parecen limitarse a ofrecer información que no va más allá de lo puramente terminológico y no siempre terminan por adecuarse a las necesidades del traductor en formación ni cubrir, en su totalidad, el área de especialización del encargo. Ante este panorama, tenemos la convicción de que cabe hacer alguna propuesta de mejora, al menos en el terreno de la traducción francés-español de cuentas anuales.

Para empezar, los recursos reseñados por los traductores en formación en sus informes finales dan a entender que no parece que haya una fuente terminológica especializada en contabilidad (francés y español) común para todos los traductores en formación. De hecho, el único recurso terminológico que prácticamente todos mencionan es la base de datos terminológica IATE, que, recordemos, no es específica de la contabilidad. En este sentido, creemos que la elaboración de un recurso terminológico (preferentemente digital, dadas las posibilidades de uso que ofrecen los sistemas de recuperación de datos) especializado en el ámbito contable y adaptado para traductores de francésespañol podría ayudar, si cabe, a mejorar la cobertura que actualmente tiene esta especialidad en esta combinación lingüística.

Por otro lado, cabría plantearse la necesidad de que la microestructura de este recurso terminológico contuviera una serie de informaciones adaptadas a las necesidades del traductor, como las que, sin ánimo de ser pretenciosos, proponemos a continuación:

- Información de tipo conceptual: en el marco de un recurso terminográfico especializado en contabilidad, quizá fuera de interés incluir no solo información sobre el árbol nocional que pudiera elaborarse dentro del campo, sino también, pensando en la adecuación del recurso al contexto de trabajo, información en torno al tipo de contabilidad (contabilidad para la gestión, contabilidad financiera, etc.) o tipo de documento (balance, memoria, estado de flujos, etc.) en el que es susceptible de enmarcarse la terminología registrada.

- Información de tipo pragmático: hemos visto que los recursos estudiados, en ocasiones, ofrecen propuestas de traducción sin detallar qué uso cabe darle, lo que lleva al traductor (sobre todo cuando el recurso utilizado ofrece más de una propuesta) a investigar, de nuevo, su uso en el propio recurso, si cabe esa posibilidad, o en otras fuentes. 
En este sentido, podría ser de interés incluir informaciones contextuales que, junto con el tipo de información mencionado anteriormente, ayudaran en la toma de decisiones de traducción, por ejemplo, referidas a la variación terminológica.

- Información de tipo fraseológico: hemos visto que la información microestructural tanto de las bases de datos terminológicas como de los diccionarios especializados no contempla este tipo de información, la cual, a tenor de las consideraciones de los expertos en traducción especializada, es un elemento vital no solo para conseguir transferir la información especializada, sino también para hacerlo tal como hacen los especialistas en la lengua de llegada de la traducción. En este sentido, y teniendo en cuenta que la memoria de las cuentas anuales es un documento con abundante fraseología, parece necesario, si no imperativo, incluir este tipo de información.

\section{A modo de conclusión}

En este trabajo hemos resaltado la necesidad de seguir evaluando los recursos terminográficos de que disponen actualmente los traductores en función no solo de su adecuación a las necesidades de este colectivo, sino también de su cobertura a tal o cual área de especialización y de tal o cual combinación lingüística. En concreto, hemos estudiado una serie de necesidades terminológicas en el marco de la traducción francés-español de cuentas anuales. A partir de estas necesidades, hemos propuesto un breve análisis de una serie de recursos utilizados en dicha situación, con especial énfasis en los de tipo terminológico. Ello nos ha llevado a reseñar una serie de aspectos que, a nuestro entender, no terminan de adaptarse a algunas de las necesidades previstas. En concreto, hemos podido inferir que los recursos terminológicos no siempre tienen la cobertura necesaria para dar acceso a la totalidad de elementos lingüísticos que potencialmente pueden aparecer en contabilidad, área de especialización en la que nos hemos centrado, ni ofrecen siempre informaciones adecuadas a las necesidades del traductor. En este sentido, hemos terminado el presente trabajo haciendo una propuesta de tipo no solo macroestructural, que implica la elaboración de un recurso terminológico especializado en el área de especialización y en la combinación lingüística con la que hemos trabajado (contabilidad francés-español), sino también microestructural, que implica la inclusión de una serie de informaciones de primera necesidad para la situación de traducción estudiada.

Creemos que la materialización de estas ideas puede ser posible en el marco de la propia formación de traductores. Este colectivo, usuario directo 
del tipo de recurso propuesto, podría desempeñar las tareas de traductor-terminólogo siguiendo las pautas de un trabajo sistemático en asignaturas relacionadas directamente con nuestro estudio, tales como traducción económica y terminología, bajo la dirección y supervisión de un equipo docente.

\section{Bibliografía}

CABRÉ, Maria Teresa. (2010) "Terminology and translation." En: Gambier, Yves \& Luc van Doorslaer (eds.) 2010. Handbook of Translation Studies. Amsterdam: John Benjamins, pp. 356-365.

CAMPS I OlIVÉ, Assumpta. (1992) "Problemes recurrents en la traducció italià/ català de textos especialitzats d'economia i comerç." En: Edo Juliá, M. (ed.) 1992. Actes del I Congrés Internacional sobre Traducció. Barcelona: Universitat Autònoma de Barcelona, pp. 169-182.

DANCETTE, Jeanne. (1992) "La complexité de la langue économique et commerciale au Québec, les défis du traducteur." TET 2-3, pp. 197-210.

DANCETTE, Jeanne. (1995) "Outil terminologique et démarche traductionnel dans le domaine commercial." Babel 41:3, pp. 161-171.

Durban, Chris. (2005) "La traduction financière. Tendances pour l'avenir." En: Gouadec, Daniel (ed.) 2005. Traduction, Terminologie, Rédaction. Actes des universités d'été et d'automne 2005. Actes du colloque international juin 2005. Traduction spécialisée chemins parcourus et autoroutes à venir traduire pour le web. Paris: La maison du dictionnaire, pp. 63-70.

FRAILE VicENTE, Esther. (2007) Las expresiones idiomáticas de la economía en inglés y español. Propuesta para un correcto tratamiento terminográfico. Valladolid: Universidad de Valladolid.

Fuertes Olivera, Pedro Antonio. (2003) "La relevancia de la labor de documentación en la traducción económica." En: Castaño Miñambres, Pilar; Tobias Tilman Klinge; Luis Pérez González \& Celia Rico Pérez (eds.) 2003. La traducción y la interpretación en un entorno global. Villaviciosa de Odón: Universidad Europea de Madrid (CD-ROM).

GALleGo HeRnÁNDEZ, Daniel. (2012) Traducción económica y corpus: del concepto a la concordancia. Alicante: Servicio de Publicaciones de la Universidad de Alicante.

Gerzymish-ARBOGaSt, Heidrun. (1989) "The Role of Sense Relations in Translating Vague Business and Economic Texts.” En: Snell-Hornby, M. \& E. Pöhl (eds.) 1989. Translation and Lexicography: Papers read at the Euralex Colloquium, July 1987. Amsterdam: John Benjamins, pp. 187-196.

GÓMEZ GONZÁLEZ-JOVER, Adelina. (2007) "Léxico especializado y traducción." En: Alcaraz Varó, E.; J. Mateo Martínez \& F. Yus Ramos (eds.) 2007. Las lenguas profesionales y académicas. Madrid: Ariel, pp. 27-40. 
GOUADEC, Daniel. (1997) Terminologie E Phraséologie pour traduire. Le concordancier du traducteur. Paris: La maison du dictionnaire.

GRUPO IUlATERM. (2013) "IULA. Terminología y traducción.” En: Grupo IulaTerm. Diploma de postgrado online: Terminología y necesidades profesionales. $8^{a}$ ed. Barcelona: IULA. Universitat Pompeu Fabra. Versión electrónica disponible en intranet.

HERAS DíEZ, Florentino. (2005) Materiales para la traducción económico-financiera francés-español. Alicante: Club Universitario.

LERAT, Pierre. (1995) Les langues spécialisées. Paris: PUF. Citado por la traducción al español de A. Ribas: Las lenguas especializadas. Barcelona: Ariel, 1997.

Lobato PAtricio, Julia; Natalia Durán Cruz \& Carmen María Morón Sánchez. (2010) "La traducción (francés-español) de textos económicos de divulgación desde una perspectiva didáctica." AdVersuS 7, pp. 73-106.

Marcos Mora, María del Carmen \& Marta Gómez Martínez. (2007) "La usabilidad en las bases de datos terminológicos on line." Glossa 2/2. Versión electrónica: <http://bibliotecavirtualut.suagm.edu/Glossa2/Journal/jun2007/ La_Usabilidad_en_las_Base_de_Datos.pdf>.

Marcos Mora, María del Carmen; Bartolomé Mesa; Mireia Ortega; Hajar Benmakhlouf; Philip Dwelle; Pedro Hernández; Juan Manuel Pérez; Irene Renau; Isabel Serván; Eleni Davradou; Fernanda López; Paulo Malvar; Agustí Mayor $\&$ Albert Morales. (2006) "Evaluación de la usabilidad en sistemas de información terminológicos on line." Anuario Hipertext.net 4. Versión electrónica: <http://www.hipertext.net/web/pag268.htm>.

MAYORAl AsENSIO, Roberto. (1996) "La traducción comercial: estrategias de traducción en razón a la disponibilidad de las fuentes de referencia terminológicas y textuales." Conferencia inédita leída en el seno del V Curso Superior de Traducción Inglés/Español (Universidad de Valladolid, 1996).

Socorro Trujillo, Karina. (2004) "Un modelo didáctico para la traducción de documentos mercantiles inglés-español." En: Bravo Utrera, S. (ed.) 2004. Traducción, lenguas, literaturas. Sociedad del conocimiento. Enfoques desde y hacia la cultura. Las Palmas de Gran Canaria: Universidad de Las Palmas de Gran Canaria, pp. 245-254.

SOLÀ TEY, Magda \& Inmaculada Vilardell Riera. (2009) Introducción a la contabilidad general. Madrid: McGraw Hill.

TEBÉ Soriano, Carles. (2001) "Elementos para la representación de los conceptos especializados en bases y bancos de datos." En: Cabré Castellví, M. T. \& J. Feliu Cortès (eds.) 2001. La terminología científico-técnica: reconocimiento, análisis y extracción de información formal y semántica. Barcelona: IULA, pp. 203-208. 


\section{Recursos}

ActufinAnCE: Les coulisses de la finance: Actualité de la finance et de l'économie. Sitio web disponible en <http://www.actufinance.fr/>.

BOE (2007): REAL DECRETO 1514/2007, de 16 de noviembre, por el que se aprueba el Plan General de Contabilidad.

Campos PlazA, Nicolás; Jesús Cantera Ortiz de Urbina; Antonio Manuel García Calero; María Dolores Espinosa Sansano \& Emilio Ortega Arjonilla. (2005) Diccionario jurídico-económico: francés-español, español-francés. Granada: Comares.

CNMV: Sitio web de la Comisión Nacional del Mercado de Valores disponible en $<$ http://www.cnmv.es/>.

COMISIÓN EUROPEA: IATE. Interactive Terminology for Europe - The EU's multilingual term base. Disponible en <http://iate.europa.eu>.

ECO-FinANZAS: Diccionario Economía - Administración - Finanzas - Marketing. Disponible en <www.eco-finanzas.com/conceptos.htm >.

ECONOMIA48 (2012): La gran enciclopedia de economía. Sitio web disponible en <http://www.economia48.com>.

EDUBOURSE: Lexique de la Bourse. Disponible en <www.edubourse.com/lexique/ rnpg.php>.

ElosuA, Marcelino (ed.) (2007) Diccionario LID multilingüe de la empresa y economía. Madrid: LID Editorial.

FUnDACIÓN PREMYSA: Glosario de términos del área económico-financiera. Disponible en <premysa.org/pdf/emprendedores/GlosarioEconomico-Financiero. pdf $>$.

NACIONES UNIDAS: UNTERM. United Nations Multilingual Terminology Database. Disponible en <http://unterm.un.org/>.

NCS SOFTWARE (2008): Sitio web sobre normas internacionales de contabilidad disponible en <http://www.normasinternacionalesdecontabilidad.es/.

PLANCOMPTABLE (2004-2009): Plan comptable générale. Nouvelles normes comptables applicables au $1^{\text {er }}$ janvier 2005. Sitio web disponible en <http:// www.plancomptable.com/>.

PONCIN YACHTS (2009): Comptes consolidés 31/08/09. Disponible en <http://www. info-financiere.fr/upload/CNS/8888/01/FCCNS025218_20100209.pdf>.

RÉPUBLIQUE FRANÇAISE: Legifrance, le service public de la diffusion du droit par l'Internet. Sitio web disponible en <http://www.legifrance.gouv.fr/>.

Termcat. Centre de terminologia: Certaterm. Disponible en <http://www. termcat.cat/>.

Unión EuROPEA: EurLex: El acceso al Derecho de la Unión Europea. Sitio web disponible en <http://eur-lex.europa.eu/es/index.htm>. 
VARIOS AUTORES (2007-2008): Dicotrec (Diccionario para traducción económica). Versión electrónica: <http://www.onyva.es/dicotrec.htm>.

\section{Agradecimientos}

Queremos manifestar nuestro agradecimiento a Rosa Estopà Bagot por sus sugerencias y comentarios sobre la primera versión de este trabajo, enmarcada en el Máster en Terminología de la Universitat Pompeu Fabra.

\section{NOTA BIOGRÁFICA / BIONOTE}

Daniel Gallego Hernández es profesor del Departamento de Traducción e Interpretación de la Universidad de Alicante. Su investigación gira en torno a la lingüística de corpus aplicada a la traducción económica, la terminología y la formación de traductores. Su tesis se titula Traducción económica y textos paralelos en internet. Imparte materias relacionadas con la traducción económica francés-español y español-francés, así como terminología. Ha publicado diferentes artículos, así como la obra titulada Traducción económica y corpus: del concepto a la concordancia.

Daniel Gallego-Hernández is a lecturer in the Department of Translation and Interpreting of the University of Alicante in Spain. His research interests include corpus linguistics applied to business translation, terminology, and translation teaching. His PhD was entitled Business translation and parallel texts on the internet. He teaches business translation between French and Spanish as well as terminology at his faculty. He has published individually various articles, and a book entitled Traducción económica y corpus: del concepto a la concordancia. 